\title{
Aircraft seating comfort: the influence of seat pitch on passengers' well-being
}

\author{
Florian Kremser, ${ }^{\mathrm{a},}$, Fabian Guenzkofer ${ }^{\mathrm{a}}$, Claudia Sedlmeier ${ }^{\mathrm{a}}$, Olaf Sabbah ${ }^{\mathrm{b}}$ and Klaus Bengler ${ }^{\mathrm{a}}$ \\ ${ }^{a}$ Institute of Ergonomics, Technische Universität München, Boltzmannstr. 15, D-85747 Garching, Germany \\ ${ }^{\mathrm{b}}$ RECARO Aircraft Seating GmbH \& Co.KG, Daimlerstr. 21, D-74523 Schwaebisch Hall, Germany
}

\begin{abstract}
One of the most important factors influencing aircraft seating comfort in economy class, is legroom. In an airline interior mock up, with the ability to adjust the seat pitch in a range of 28 inches to 43 inches, a study to investigate the influence of seat pitch on passengers' well-being was conducted. In a pre-study, aspects of subjective postural sensations and spatial perception were identified, and a questionnaire was developed. In the main study, 30 subjects rated at different seat pitch settings the spatial perception and the ability of adopting and changing sitting postures. As a result a functional relationship between overall well-being, the subjects' anthropometry and seat pitch was developed. Furthermore it was identified, that there is a maximum overall well-being at a seat pitch of 34 inches to 40 inches, depending on the passengers' anthropometry. A further enlargement of seat pitch, led to a reduction of well-being.
\end{abstract}

Keywords: seat pitch, legroom, spatial perception, sitting posture, economy class

\section{Introduction}

Over the years, commercial air traffic and the number of passengers have been constantly increasing. One reason for this development is the increase of low cost carriers (LCC) [3]. The lower prices of LCCs compared to full service carriers (FSC) are often accompanied with a reduction of comfort and service. FSCs have the possibility to set themselves apart by offering a higher comfort level to their passengers.

Comfort is not simply the absence of discomfort, and indeed both can occur at the same time [12]. A cluster analysis identified that comfort is mainly associated with pain and biomechanical factors. Comfort has also been identified as associated with relaxation, luxury and well-being [12]. Vink [10] explains the following inputs as factors influencing comfort and discomfort:

- History

- State of mind

- Visual input

- Environmental factors (e.g. smell, noise, temperature and humidity)
- Pressure distribution

- Posture and Movements

A survey [7] conducted in 1975 pointed out that passengers rated seat comfort as the most important factor influencing discomfort, followed by noise and temperature. Furthermore in a 1999 accomplished study [5], 930 passengers evaluated several, their seats concerning factors. Legroom $(15.7 \%)$, back support (10.2\%) and head support (9.7\%) were among the five factors rated poor or very poor by the highest percentage of surveyed passengers.

Vink [10] figured out that legroom has a very high influence on aircraft interior comfort. That was shown by a correlation coefficient of .72 between legroom and comfort.

The available legroom and space for passengers are mainly influenced by seat pitch. In Figure 1 the measures seat pitch (A) and legroom (B) are shown. Seat pitch is defined as the distance from a point on the seat in one row to the same point on a seat in the next row. The depth and the contour of the backrest reduce seat pitch to the available legroom (B) $[6,10]$. Bauch [2] defined the measures clearance, width, and,

*Corresponding author. E-mail: kremser@tum.de 
seat pitch, as comfort measures, and stated that maximum comfort is reached at the maximum values of these comfort measures.

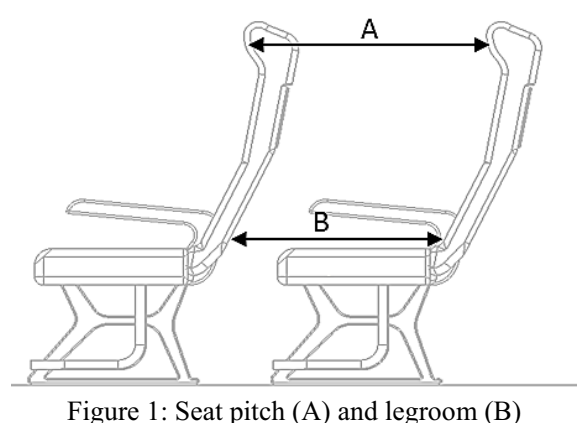

A survey conducted in 2009 established, that passengers would pay up to $120 €(170 \$)$ for higher comfort and up to $33 €(47 \$)$ for a larger seat pitch [1]. To maximize the number of passengers on board of LCCs the seat pitches are often smaller than on board of FSCs. There are even thoughts about establishing standing room instead of seats on short haul flights. Even if leisure travelers show no real willingness to pay higher prices for better service, business travelers do [4]. This is one of the interests in establishing an economy plus class. Such a class, between economy and business class, offers primarily a larger seat pitch to the passengers than standard economy class.

To identify the seat pitch of maximum comfort for economy class travelers, a mockup study is conducted. The objective of this study is to assess the influence of seat pitch on comfort factors and overall well-being, dependent on the passengers' anthropometry.

\section{Materials and Methods}

\subsection{Test setup}

For the subject-based study an aircraft interior mock-up was built. It consisted of the interior parts of an Airbus A340 and two short haul seating rows provided by RECARO Aircraft Seating (Figure 2). To be able to adjust the seat-pitch easily during the experiments, the front row was mounted on linear slides. The seat pitch was continuously variable from 28 in. to 43 in. This range covers typical economy class and narrow business class cabin layouts [3]. The rear seating row was fixed rigidly. The whole mock-up had an inclination of $3^{\circ}$; this is a typical angle of the aircraft cabin during stationary cruising flight.

For the reconstruction of subjects' sitting postures and for having the possibility of a qualitative evaluation of the sitting behavior, a multi-camera system was installed. The cameras were mounted in a way that made it possible to track each subject by at least 2 cameras at the same time.

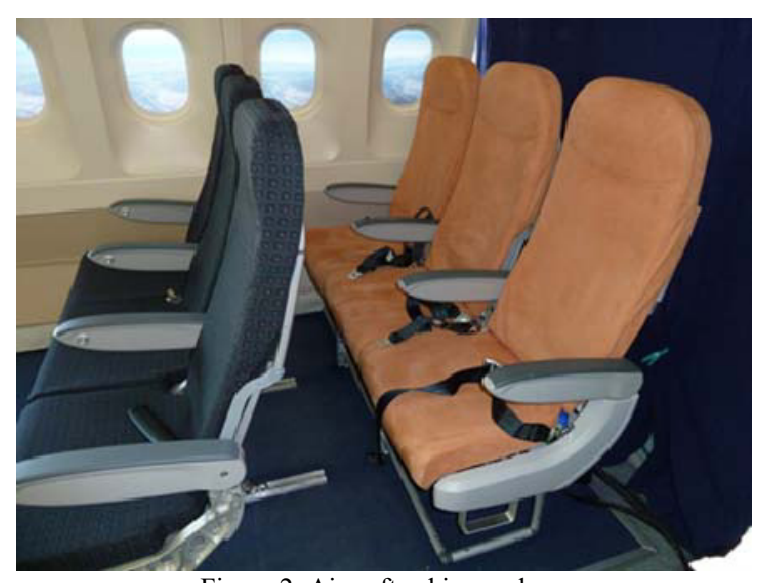

Figure 2: Aircraft cabin mock-up

\subsection{Experimental Design}

The study was divided into a pre-study for the development of a proper questionnaire and a main study.

\subsubsection{Pre-study}

The objective of the pre-study was to develop a paper-based questionnaire to quantify the influence of different seat pitches on the passengers' wellbeing.

In the pre-study, 8 subjects ( 7 male, 1 female; no exclusion criteria) sat down in the middle seat of the rear seating row. They were asked to verbally express the factors influencing their well-being and comfort sensations for different seat pitches.

Finally, their answers led to the following ten expressions which were integrated into the questionnaire of the main study. These statements can be clustered in four categories:

Postural sensation:

- I can easily adopt a comfortable sitting posture

- I can change easily from one sitting posture to another 
Spatial perception:

- I feel restricted

- I feel restricted by the seat-width

- I feel restricted by the distances of the armrests

- I feel restricted by the distance of the seating rows

- I feel restricted by my seatmate

- I feel like sitting in front of a wall

Privacy:

- I feel lost because of the distance of the seating rows

Present mood:

- I feel stressed out because of the distance of the seating rows

\subsubsection{Main study}

During the main study, the subjects rated their subjective feelings/impressions for different seat pitches (Table 1). The evaluation process was divided into two steps. First, subjects rated on a five point Likert Scale, from 1 (strongly disagree) to 5 (strongly agree), how well the phrases, created in the pre-study, applied to the present scenario. Second, subjects rated their overall level of well-being on a scale from 1 (lowest level of well-being) to 10 (highest level of well-being).

The simulated seat pitches are shown in Table 1. From an anthropometric point of view, the seat pitch of scenario $\mathrm{A}$ is the absolute minimum recommended by Quigley et al. [6]. The seat pitch of scenario $\mathrm{C}$ is the average seat pitch of the economy class on short haul flights. The seat pitch of scenario $\mathrm{D}$ is the maximum seat pitch provided by some airlines in the economy class on short haul flights. The seat pitch of scenario E shows a typical value for the economyplus class. Scenario $G$ is the upper limit that can be realized in the mock-up. Scenarios B and F are intermediate values to ensure a more accurate regression. The sequence of the scenarios was randomized, to reduce the possibility of fatigue and boredom effects. Furthermore the seat position (window, middle or aisle) was randomly assigned to the subjects.

Table 1

Seat pitches and legroom of the test scenarios

\begin{tabular}{lll}
\hline Scenario & $\begin{array}{l}\text { Seat pitch } \\
\text { (inch) }\end{array}$ & $\begin{array}{l}\text { Legroom } \\
\text { (inch) }\end{array}$ \\
\hline $\mathbf{A}$ & $28^{\prime \prime}$ & $26^{\prime \prime}$ \\
$\mathbf{B}$ & $30^{\prime \prime}$ & $28^{\prime \prime}$ \\
$\mathbf{C}$ & $32^{\prime \prime}$ & $30^{\prime \prime}$ \\
\hline
\end{tabular}

\begin{tabular}{lll}
\hline D & $34 "$ & $32 "$ \\
E & $36 "$ & $34 "$ \\
F & $40^{\prime \prime}$ & $38^{\prime \prime}$ \\
G & $43 "$ & $41 "$ \\
\hline
\end{tabular}

In the main study, the subjects' anthropometry, the seat position (window, middle or aisle) and the seat pitch were independent variables. The subjective rated level of well-being and the level of acceptance of the phrases developed in the pre-study were dependent variables.

\subsection{Subjects of the main study}

Thirty subjects participated in the main study, 17 male and 13 female. There were no restrictions in size or age for the selection of the subjects (Table 2).

Table 2

Mean and standard deviation of age, stature and buttock-kneelength of the participating subjects sorted by sex.

\begin{tabular}{lll}
\hline & Male & Female \\
\hline $\mathbf{n}$ & 17 & 13 \\
\hline Age (years) & $32.9 \pm 12.5$ & $30.3 \pm 10.4$ \\
\hline $\begin{array}{l}\text { Stature (cm) } \\
\text { Buttock to } \\
\text { knee length } \\
\text { (cm) }\end{array}$ & $180.3 \pm 7.6$ & $167.9 \pm 7.7$ \\
$\begin{array}{l}\text { Eye height } \\
\text { sitting (cm) }\end{array}$ & $81.9 \pm 4.2$ & $76.7 \pm 1.9$ \\
\hline
\end{tabular}

\subsection{Experimental Procedure}

Three subjects participated one experimental run as a group.

In the beginning of the experiment, the subjects had to complete a demographic questionnaire. Furthermore, the following anthropometric measures of each subject were acquired:

- stature

- $\quad$ sitting height

- $\quad$ eye height sitting

- acromial height sitting

- elbow height sitting

- knee height sitting

- $\quad$ buttock to knee length 
- hip width

- shoulder width

Next, the subjects were advised to sit in their assigned seats and to fasten their seatbelts in the same way they would during a stationary cruising flight and to additionally set the backrest to an upright position.

During the next 30 minutes the seat-pitch was varied in the six discrete steps (see Table 1) in a randomized order. After changing the seat pitch the subjects evaluated their possibilities to adopt and change sitting postures, the spatial perception and their overall well-being.

\section{Results}

To explore the data, standard statistical methods were used. The data analysis was conducted using SPSS 19 and MATLAB. Significances were calculated using one-way repeated-measures ANOVA. In case of violating the assumption of sphericity, the Greenhouse-Geisser correction was used.

\subsection{Influence of seat pitch on subjective perception of sitting posture}

As shown in Figure 3 it is easier for the subjects to adopt a comfortable sitting posture or to change their posture with a growing seat pitch. Beginning at a seat pitch of $28 \mathrm{in}$. the ability to adopt a comfortable posture and to change ones posture is increasing. At a seat pitch of 36 in., saturation of the dependent variables is reached, even at larger seat pitches there is no change in the asked factors.

The results show that the easiness of adopting a comfortable sitting posture is significantly influenced by the seat pitch, $F(2.57,74.55)=16.51, \mathrm{p}<.001$. Also the easiness of changing ones posture is significantly influenced by the seat pitch, $\mathrm{F}(2.58,74.85)=$ $19.86, \mathrm{p}<.001$.

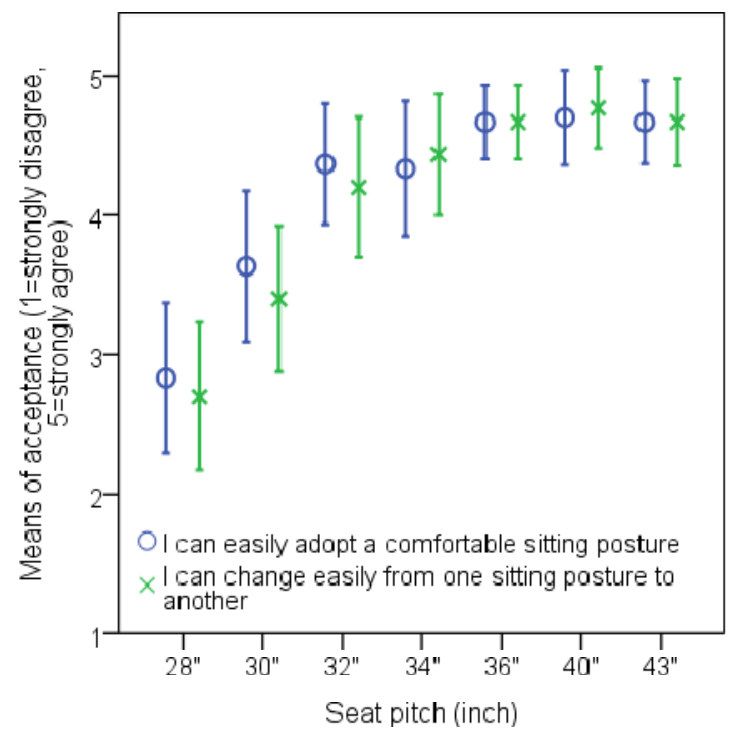

error bars: $95 \% \mathrm{Cl}$

Figure 3: Influence of seat pitch on sitting posture (1=strongly disagree, $2=$ inclined to disagree, $3=$ neither, $4=$ inclined to agree, $5=$ strongly agree)

For both variables, the enlargement from 28 in. to 30 in. and from 30 in. to 32 in. is significantly ( $p$ $<.05)$ different. For further seat pitch enlargements (32 in. to 34 in., 34 in. to 36 in., 36 in. to 40 in., 40 in. to $43 \mathrm{in}$.), the results are not significantly different ( $\mathrm{p}$ $>.05)$

Furthermore, a correlation analysis shows that the variables adopting a posture and changing postures are highly correlating $(\mathrm{r}=.89, \mathrm{p}<.001)$.

\subsection{Influence of seat pitch on spatial perception}

In Figure 4 the influence of seat pitch on spatial perception is shown. Beginning with a seat pitch of 28 in. the feeling of being restricted is decreasing towards a minimum level at around 40 in. Also the feeling of being stressed out because of the available space reaches its minimum at around $40 \mathrm{in}$. Only the feeling of sitting in front of a wall shows no asymptotic behavior and is constantly shrinking with growing seat pitch. The feeling of being lost is more or less constant towards a seat pitch of 36 in., afterwards it is rising. 


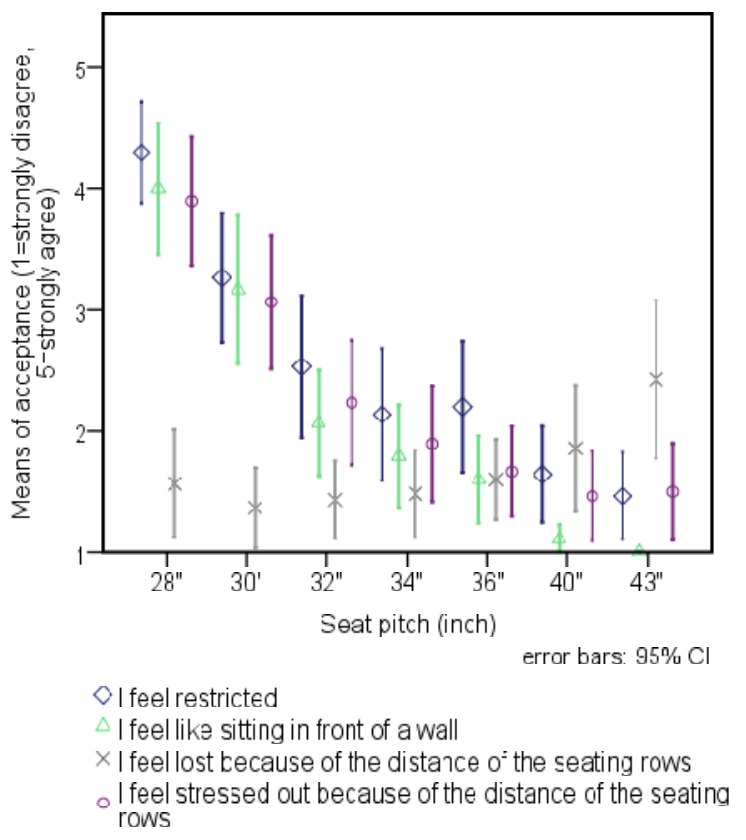

Figure 4: Influence of seat pitch on spatial perception posture ( $1=$ strongly disagree, $2=$ inclined to disagree, $3=$ neither, $4=$ inclined to agree, $5=$ strongly agree).

The feeling of being restricted is significantly influenced by the seat pitch, $F(6,162)=30.10$, p $<.001$. Furthermore, the feeling of sitting in front of a wall $(\mathrm{F}(3.74,104.63)=36.85, \mathrm{p}<.001)$ and the feeling of being lost $(F(6,168)=3.93, p<.05)$ are significantly influenced by the seat pitch. The feeling of being stressed out is not significantly affected by the seat pitch, $\mathrm{F}(1.04,30.02)=.98, \mathrm{p}>.05$.

Seat pitch enlargement induces a significant difference from $28 \mathrm{in}$. to $30 \mathrm{in}$. and from $30 \mathrm{in}$. to $32 \mathrm{in}$. for the feeling of sitting in front of a wall $(p<.05)$ and the feeling of being stressed up $(\mathrm{p}<.001)$. Further seat pitch enlargements induce no significant difference for these feelings $(p>.05)$. For the feeling of being restricted a significant difference $(p<.05)$ is induced for the seat pitch enlargement from 28 in. to 30 in., 30 in. to 32 in. and 32 in. to 34 in. A further enlargement has no significance influence on the variable $(\mathrm{p}>.05)$

\subsection{Dependence of buttock to knee length and seat pitch on passengers' overall well-being}

As shown in Figure 5, the overall well-being can be described as a function of buttock to knee length and seat pitch. At a seat pitch of 28 in. the level of overall well-being is almost the same independent of the buttock to knee length of the subjects. The maxi- mum level of overall well-being (7.14) of subjects with a buttock to knee length of $53 \mathrm{~cm}$ is reached at 38 in., for subjects with a buttock to knee length of $60 \mathrm{~cm}$ at 39 in. (well-being level: 8.30) and for subjects with a buttock to knee length of $66 \mathrm{~cm}$ the maximum level of well-being (8.93) is reached at a seat pitch of $40 \mathrm{in}$. After the maximum the level of wellbeing is decreasing.

The fitting function, a polynomial of degree 2 is shown in Eq. 1. $\left(\mathrm{R}^{2}=.45\right)$.

$w b=$ well being

$s p=$ seat pitch

$b k l=$ buttock knee length

$w b=f(s p ; b k l)$

$w b=-40.86+2.424 \times s p-0.056 \times b k l-0.037 \times$

$$
\mathrm{sp}^{2}+0.008 \times s p \times b k l-0.001 \times b k \mathrm{l}^{2}
$$




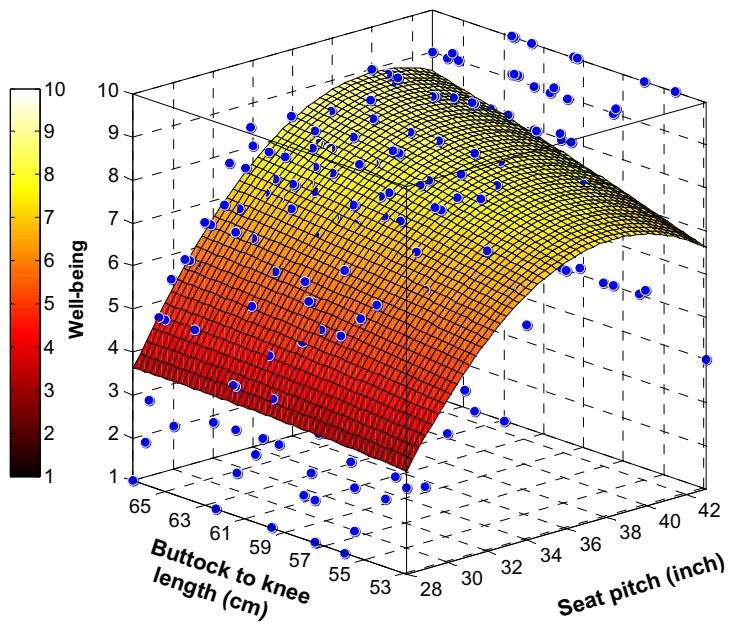

Figure 5: Surface fitting $\left(\mathrm{R}^{2}=.45\right)$ for well-being ( $1=$ lowest level; $10=$ highest level) dependent on buttock to knee length and seat pitch.

\subsection{Dependence of eye height and seat pitch on passengers' overall well-being}

In Figure 6 one can see the relationship of eye height, seat pitch and overall well-being. The minimum overall well-being level of 2.10 is located at a seat pitch of $28 \mathrm{in}$. for subjects with an eye height of $92 \mathrm{~cm}$. Furthermore, subjects with a medium eye height rated the level of well-being at a seat pitch of 28 in. higher (e.g. eye height: $82 \mathrm{~cm}$, well-being level: 4.10) than subjects with a smaller eye height (e.g. eye height: $73.4 \mathrm{~cm}$, well-being level: 3.24$)$. The maximum level of overall well-being (6.83) for subjects with a small eye height is reached at a seat pitch of 38 in. With an increasing seat pitch, the level of wellbeing decreases. This effect can also be seen for subjects with a medium eye height (eye height: $82 \mathrm{~cm}$; maximum well-being: 8.96; seat-pitch: 40in.) and for subjects with a larger eye height (eye-height: $92 \mathrm{~cm}$; maximum well-being: 8.80; seat-pitch: $42 \mathrm{in}$.).

The fitting function, a polynomial of degree 2 is shown in Eq. $2\left(\mathrm{R}^{2}=.45\right)$.
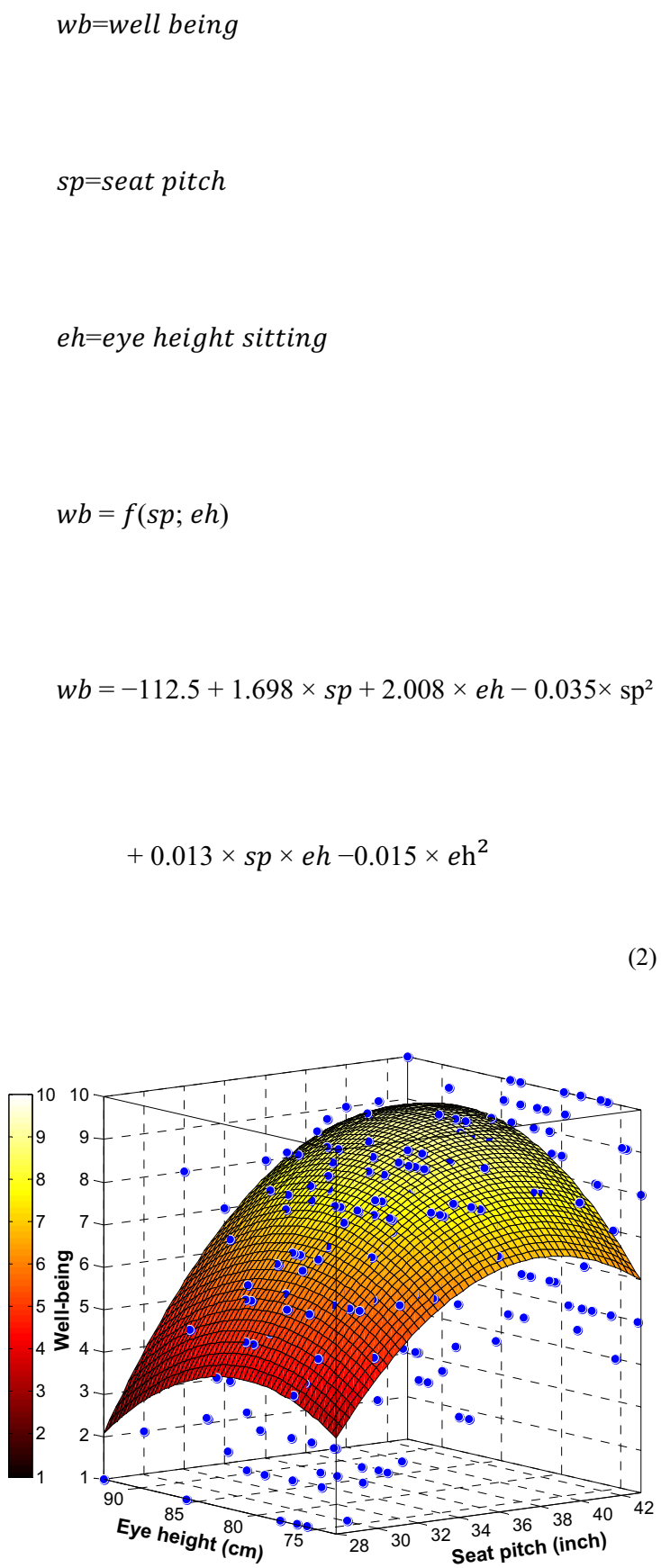

Figure 6: Surface fitting $\left(\mathrm{R}^{2}=.45\right)$ for well-being ( $1=$ lowest level; $10=$ highest level) dependent on eye-height-sitting and seat pitch. 


\section{Discussion}

The overall well-being of a passenger is influenced by physiological and psychological factors [9] [1011]. In this study, an important influence on comfort was induced by the physiological factors of adopting and changing postures. But these two variables are highly correlated $(\mathrm{r}=.89, \mathrm{p}<.001)$ so, in future studies, it would not be necessary to ask for both factors. It would be sufficient to simply ask the subjects to rate the easiness of changing their posture because a comfortable sitting posture is only comfortable until another more comfortable posture is adopted. Furthermore, the possibility to change ones posture easily, could lead to the effect that passengers do not sit too static on an airplane. This will have positive effects on the risk of thrombosis.

The psychological aspects are mainly affected by visual perception [10]. The variables of feeling stressed out and of feeling like sitting in front of a wall are also high correlating $(\mathrm{r}=.79, \mathrm{p}<.001)$. Therefore, in future studies, there would be no need for asking the subjects both factors.

The main result of this study is the quantification of the influence of seat pitch on the passengers' wellbeing. A key issue is the identification of the seat pitch of maximum overall well-being on short haul flights. It was discovered that comfort and acceptance is not simply growing with a larger seat pitch, but there is a turning point where larger seat pitches lead to less well-being. So a maximization of the comfort measures defined by Bauch [2] does not automatically result in maximum passenger comfort.

The optimal seat pitch is, on the one hand, basically influenced by the buttock-knee length $\left(\mathrm{R}^{2}=.45\right)$. That relationship was expected from the beginning of the study, because the effective legroom is shrinking with a larger buttock to knee length. Furthermore, for subjects with a larger buttock to knee length, the ability to adopt a comfortable posture is reduced.

Furthermore the influence of eye height on passengers' overall well-being was shown. A larger eye height is equal to a larger torso length. A reason for the lower well-being of subjects with smaller and taller torsos compared to subjects with a medium torso length could have two different reasons. Spatial perception of subjects with a lower eye height might be dominated by the feeling of being restricted, because they had no possibilities to watch over the backrests of the sitting row in front. Subjects with a medium eye-height had the possibility to look over the seat row to their front. Because of the correlation of leg length and torso length, these subjects had also the possibility to sit in a more comfortable position and to change their sitting postures more easily. Subjects with a larger eye height had a clear view over the front sitting row but were restricted by their longer legs.

Comparing the results of posture and spatial perception with the results of overall well-being, the seat pitch for maximum well-being ranges from $34 \mathrm{in}$. to 42 in. (legroom 32 to 40 in.), depending on the passengers anthropometry.

\section{Acknowledgements}

The authors thank RECARO Aircraft Seating $\mathrm{GmbH}$ for the support in this research project.

\section{References}

[1] Balcombe, K., Fraser, I., \& Harris, L. (2009). Consumer willingness to pay for in-flight service and comfort levels: A choice experiment. Journal of Air Transport Management, 15(5), 221-226.

[2] Bauch, A. (2001). Ergonomie in der Flugzeugkabine - Passagierprozesse und manuelle Arbeitsabläufe. In D. Scholz (Ed.), Flugzeugkabine, Kabinensysteme - die nächsten Schritte. Workshop DGLR-Fachbereich T8 "Flugzeugbordsysteme", DGLR-Fachausschuß S2.1 "Starrflügelsysteme", Airbus Conference Center, Hamburg-Finkenwerder, 10./11. Mai 2001; Tagungsband (pp. 49-57). Bonn: DGLR.

[3] Brundrett, G. (2001). Comfort and health in commercial aircraft: a literature review. In: The Journal of the Royal Society for the Promotion of Health 121 (1), S. 29-37.

[4] Lee, D., \& Luengoprado, M. (2004). Are passengers willing to pay more for additional legroom? Journal of Air Transport Management, 10(6), 377-383.

[5] Pierce, W. et al. (1999). Air Quality on Commercial Aircraft. In: Ashrae Journal September 1999, S. 26-33.

[6] Quigley, C., Southall, D., Freer, M., Moody, A., \& Porter, J. M. (2001). Anthropometric study to update minimum aircraft seating standards. $\quad$ Retrieved from http://hdl.handle.net/2134/701.

[7] Richards, L. G., \& Jacobson, I. D. (1975). Ride Quality Evaluation 1. Questionnaire Studies of Airline Passenger Comfort. Ergonomics, 18(2), 129-150.

[8] Richards, L. G., \& Jacobson, I. D. (1977). Ride Quality Assessment III: Questionnaire Results of a Second Flight Programme. Ergonomics, 20(5), 499-519.

[9] Shen, W., \& Parsons, K. C. (1997). Validity and reliability of rating scales for seated pressure discomfort. International Journal of Industrial Ergonomics, 20(6), 441-461.

[10]Vink, P., Brauer K. (2011). Aircraft Interior Comfort and Design. Boca Raton, FL: CRC Press

[11] Vink, P. (2005). Comfort and design: Principles and good practice. Boca Raton: CRC Press.

[12]Zhang, L., Helander, M.G., Drury, C.G. (1996). Identifying factors of comfort and discomfort in sitting. Human Factors, 38(3): 377-389 